\title{
Rejection of Good Manuscripts: Possible Reasons, Consequences and Solutions
}

Ahmed Ibrahim Fathelrahman*

Assistant Professor, Department of Pharmacy Practice, College of Pharmacy, Qassim University, Saudi Arabia

*Corresponding author: Ahmed Ibrahim Fathelrahman, Assistant Professor of Pharmacy Practice, College of Pharmacy, Qassim University, P.O. Box 6800, Buraidah, Saudi Arabia, Tel: 00966547754981; E-mail: afathelrahman@yahoo.com

Rec date: Oct 30, 2014, Acc date: Jan 02, 2015, Pub date: Jan 08, 2015

Copyright: (c) 2015 Fathelrahman Al. This is an open-access article distributed under the terms of the Creative Commons Attribution License, which permits unrestricted use, distribution, and reproduction in any medium, provided the original author and source are credited.

\begin{abstract}
This paper describes various aspects related to manuscript rejection particularly those manuscripts proved later on to be good and explains efforts made by some biomedical journals to reduce the loss in the scientifically sound works because of rejection. Rejection of good manuscripts is a complex issue and requires initiating further discussions to encourage the emergence of new and widely applicable solutions. Studying this issue or writing about it would help beginners who seek publishing their data but face difficulties solve the problems of rejection and getting their work published in a reasonable time frame. Also, appreciating that manuscript rejection is a common occurrence would help relieving the symptoms of frustration and reduce feelings of disappointment.
\end{abstract}

Keywords: Manuscript rejection; Quality of publications; Peer review

\section{Introduction}

Manuscript rejection is a hurting and a disappointing experience especially when it is repeated several times. Colleges and universities always evaluate and promote the academic staff based on their number of publications. Manuscript rejection represents a major barrier for junior staff to get promoted and develop their carrier especially that the rates of manuscripts' rejection in the high impact journals reach as much as $90 \%$ [1]. An author who target publication in high prestigious journals may spend many years to publish just small parts of his research findings. This had lead many of the researchers and academic staff particularly in developing countries to publish their studies in local and low ranked journals.

Several studies attempted to address the reasons of manuscript rejection by biomedical journals. Studying this issue or writing about it would help beginners who seek publishing their data but face difficulties solve the problems of rejection and getting their work published in a reasonable time frame. Also, appreciating that manuscript rejection is a common occurrence would help relieving the symptoms of frustration and reduce feelings of disappointment. Common reasons included lack of the importance and relevance of the subject matter; lack of consistency between study design, results, and conclusions; lack of originality; and inappropriate, questionable or flawed methodology [2-4]. In many situations different journals send the same manuscript to the same reviewers and unless a significant change in the manuscript has been made, reviewers would recommend "rejection" again. This explains partially the experiences of frequent rejections of a particular manuscript when an author keeps resubmitting it to several journals as it was written firstly and without making any effort to modify it. As a manuscript is getting rejected several times data becomes out-of-date. This provides another reason to reviewers and editors for rejection. Rejection because the data is getting out-of-date occurs in researches related to some fields such as epidemiology and clinical works and those studies which are surveybased but may not be seen in laboratory-based researches as such data can be published at any time as long as there is an interest in the findings.

However, there is some evidence that not all rejected manuscripts are of a poor quality. According to Woolley \& Barron, at least $50 \%$ of rejected manuscripts are published within 2 years after being rejected although this period may reach up-to 5 years [1]. According to Calcagno and associates, published papers that have a history of prior rejection are cited more than those which do not have such history [5]. This supports the notion that many rejected papers were actually good or at least having potentiality to become so. Then, why some good papers are getting rejected? Other factors beyond those identified by previous studies might be playing role in the rejection of the good work. The present paper discusses various aspects related to the rejection of good quality manuscripts and describes efforts been made by some biomedical journals to reduce the loss in the scientifically sound works due to rejection.

\section{The poor quality and insufficient reviewing}

Basically, the peer review exercise improves the quality and the scientific value of the papers published in biomedical journals [1]. Consistent with this, the finding by Calcagno and associates [5] supports the role of the peer review in increasing the appeal and the value of the initially rejected manuscripts. This is because the quality of those papers is improved further upon repeated peer review. Nevertheless, some manuscripts with good potentialities might be rejected because of a poor reviewing.

Besides assisting the editors to make decisions on manuscripts, peer review is supposed to help authors improve their research reporting. Unfortunately, some reviewers provide only few comments that may even be too general and of little value. Poor review reporting affects the quality of the published science. According to Murray and colleagues, substantial proportions of randomized trials related to cancer research between 2002 and 2006 suffered from methodological problems [6] Kelly et al. found that many of the systematic reviews published in the emergency medicine literature contain major flaws [7]. It is possible that due to work load some reviewers are not able to read the manuscripts that they are reviewing very well. Norman linked 
poor quality of review with time pressure and lack of rewards for good peer reviewing [8]. It is always an ethical obligation that reviewers spend enough time on reading the manuscript that they are reviewing, and accordingly providing thorough and high quality comments. Poor reviewing results in poor quality publications. In the same time, a reviewer who does not read well; who does not understand important aspects related to the study and the interpretation of the findings; who provides a poor quality report; is more likely to recommend rejection.

Another possible reason for a poor reviewing is a misunderstanding of the study design and findings emerges due to differences between the area of expertise of the reviewer and the field of study of the particular paper. This is because what is deemed to be not accepted of methods and terminologies in a certain field of research may be accepted and widely used in another field. This is like a reviewer with a background in clinical research who is evaluating a paper related to the public health and vice-versa. Some journals may select reviewers based on their publications in the particular area which is not importantly reflecting their actual field of experience. Personally I have seen some academic staff with a background in microbiology or pharmacology, as example, supervises student whose research in the social and community based areas.

\section{Reviewers' overvaluation of criticism for its own sake and the lack of flexibility}

Some good research maybe getting rejected due to some incorporated limitations such as small samples sizes in cases of very rare occurrences and loss to follow-up in very large surveys although the presence of such limitations does not importantly imply a major flaw in the methodology. However, a research limitation should not be ignored by the researcher; rather it should be raised and disclosed in a transparent fashion. Findings should be discussed and interpreted on view of such limitations. Reviewers should differentiate and try to make a balance between what is ideally supposed to be done in normal situations and what a researcher actually was able to do according to the available resources and on view of the scope of the study. Norman described a review culture occurs among some reviewers that encourages "Overvaluation of criticism for its own sake, and inappropriate statistical dogmatism" [8]. He mentioned that some reviewers are having tendency to criticize the choice of statistical methods based on statistical assumptions that could in fact be flexibly violated without significantly affecting the validity of findings. Dutta pointed out a type of reviewers who engage in reviewing activities as if they are involved in some sort of fighting or considering the review an opportunity for showing muscles and power [9]. In contrast to the reviewers who make insufficient poor quality reviews, this kind of culture is found among reviewers who wrote very lengthy and much detailed reports. In this case, a reviewer is making efforts that are more than required. Then, he is not accepting any excuse from the side of the authors whenever finds anything that seems to be not in the appropriate place.

Dutta suggested Ten Commandments to improve the reviewing capacities of the reviewers [9]. Among those, he advised reviewers to approach reviewing as a collaborative task rather than a struggling war, avoid turning it into a muscle show to exercise personal knowledge and abilities, being reflexive, understanding the paradigms, understanding the limitations of the project, and being specific in giving recommendations.
In general, it is important that a reviewer provides comments that are specific and shows the level of importance of the particular comment. Some journals used structured forms for providing reviews and ask reviewers to rate the significance and urgency of their comments and recommendations as optional or obligatory.

\section{Editors' obligations and their limitations as human beings}

We cannot totally rule out the role of some editors or that of the peer review system in rejecting good papers. In many occasions, the result of the peer review and the decision taken by an editor comes with a surprise to the authors as well as to some reviewers. Richard Smith (an editor of the BMJ and chief executive of the BMJ Publishing Group for 13 years) said "I regularly received letters from authors who were upset that the BMJ rejected their paper and then published what they thought to be a much inferior paper on the same subject" [10]. An author of two different papers knows the value of his papers more than anyone else, at least which one of two papers is more important. This is may be due to some subjectivity in the peer review of some journals and may be because some editors pick up what seems to them more interesting according to the journal scope. Personally it seems to me that there might be situations where some editors establish tendency to accept or reject a particular manuscript even before sending it to the peer review. In the same context, Smith showed that in most situations decisions established through peer review are coming in concordance with the editors' prior judgments [10]. This provides some evidence that the journal policy and the peer review system affects both editors and reviewers. Smith highlighted "Maybe a lone editor, thoroughly familiar with what the journal wants and knowledgeable about research methods, would be enough" [10].

A question that worth being raised, which kind of review reports affect an editor more while taking a decision on a manuscript, the detailed lengthily written or the concisely shortly written one? May be this would be a good research question for a future study. Anyway, editors should not be affected much by the length or the depth of the review report and how much critics are received. Many critics and raised issues are not importantly implying a weakness in the manuscript. A reviewer may provide much more insights into the topic that he finds more interesting, clearly written and methodologically well designed.

\section{The constraints of the journals' style, the peer review system and the readers' expectations}

In fact, it is difficult to separate between an editor as a human being at one hand and the journals' peer review as a system for manuscripts' evaluation on the other hand. This is because the system adopted by each journal for the peer review shapes and influences the decisions taken by the editors. It is not a secret that some limitations and some sources of bias in peer review have been identified by some researches or highlighted by some experts [10]. Richard Smith concluded "In addition to being poor at detecting gross defects and almost useless for detecting fraud it is slow, expensive, profligate of academic time, highly subjective, something of a lottery, prone to bias, and easily abused" [10]. Smith described some examples of bias in beer review process such as bias against authors from less prestigious institutions, and bias against negative studies. Some evidence showed that a manuscript is more likely to be accepted if it is originating from the same country as that of the publishing journal [2,11]. Many manuscripts that report well designed and scientifically sound research are being rejected without a justified opinion except that they are 
found to be not interesting. May be because the findings are negative or not new, or the topic is not a hot topic according to the scope of the journal.

However, the present paper is not intended to blame the editors or the journals' review system. We admire that this is a multi-factorial issue. The limited space for publishable articles in biomedical journals represents a constraint for publishing every good work. The scope of the journal and the interest and expectations of the readers of those journals represent another constraint that we cannot ignore. Woolley \& Barron declared that "many editors who receive more submissions than they can accept have to reject good manuscripts" [1].

\section{Rewarding the reviewers as a motivation to improve their reviewing}

As shown earlier, the reviewers represent an essential part in improving the quality of the published science either directly when a manuscript is accepted and being published during the same submission and after making the required corrections or indirectly when an author is re-submitting the work to another journal and is making use of the comments and critics provided during an earliest review. Accordingly, reviewers deserve getting some credits on that. This does not imply that reviewers should be paid for reviewing jobs. Currently, the rewards provided to the reviewers are limited, may be being offered a free access to the publisher's materials or being acknowledged by the journal. As a solution, some open accessed journals have made a good initiative towards acknowledging the intellectual contribution that reviewers made in improving the value of the published research and science via publishing in their websites what is called "pre-publication history" a section that includes the names of the reviewers who evaluated the paper together with their comments and critics. Smith mentioned rewarding the reviewers among options to improve peer review [10]. It is possible to establish a system that evaluate scientists and researchers based on their contribution to the peer review taking into consideration the quality and the frequency of reviewing activities and the rank of the journals to which review reports are made. The system could be established in conjunction with the system in the universities that is used to promote academic faculty members. This is like considering additional points for promotion based on reviewing activities.

\section{The Open Access and the publication of studies with negative results improve the capacity of manuscripts acceptance}

To reduce the severe loss in good publications there is a need to increase the capacity of possibly publishable papers. The scientific community recognized the importance of publishing studies that show negative results as well as those show positive findings when the concept of evidence based practice became dominant and it became evident that it is important to reduce publication bias as so as to get a comprehensive overview of the literature to perform systematic reviews and meta-analyses that make valid conclusion about the effectiveness of treatments, policies and programs.

Open access journals represent a solution in this regards and during the last few years many known publishers started opening new journals which are open accessed such as "BMJ Open", "Sage Open" and "Springer Plus". This is because besides being freely accessed, open access journals also accept unlimited number of papers. Moreover, to reduce the loss in the scientifically sound works some publishers initiated journals which focus only on publishing the papers that face difficulties due to negativity of findings or because of the readers' limited interest. This is like "BMC-Research Notes" and the "Journal of Negative Results in BioMedicine".

\section{Conclusion}

This paper discussed various aspects related to manuscript rejection. Rejection of good manuscripts is a complex issue and requires opening the door for further discussions, and elaborations between publishers, editors, reviewers and the wide scientific community. Such communication would aid in the continuous improvement in the peer review systems and encourages the emergence of new initiatives and widely applicable and fair solutions.

\section{Competing Interests}

The author declares no competing interest.

\section{Authors' Information}

Assistant Professor, Department of Pharmacy Practice, College of Pharmacy, Qassim University, Saudi Arabia. The author got some experience as an author of a group of articles published in a number of biomedical journals besides an experience as a reviewer.

\section{Summary}

Rejection of good manuscript has bad consequences on the scientific literature. To solve the problem of unnecessary rejection and improve quality of submissions the following advises may help:

\section{Authors}

- From the beginning, choosing the relevant journal for submission based on the journals' scope and the characteristics, expectations and interest of the readers

- Upon rejection, trying making use of the suggestions provided by the reviewers and if needed re-structuring papers according to reviewers critics before considering resubmission to another journals.

\section{Reviewers}

- Being flexible while evaluating the merit of a paper and trying to keep a balance between the importance of the studied topic, the appropriateness of the used methods, the importance of findings and the limitation of the overall work.

- Being objective and specific while providing feedback and avoiding making general negative comments (some journals use structured format for providing a review report)

\section{Editors}

- $\quad$ Selecting the appropriate reviewers for the particular paper as inappropriate selection leads to either acceptance of a low quality paper or rejection of a good paper.

- If a rejection decision must be made based on a lack of interest and due to readers expectations it would be good to transparently refer to this to help the authors make the right decision regarding future submissions or resubmission of a rejected paper. 
Citation: Fathelrahman Al (2015) Rejection of Good Manuscripts: Possible Reasons, Consequences and Solutions. J Clinic Res Bioeth 6: 204. doi:10.4172/2155-9627.1000204

Page 4 of 4

- If the manuscript is not fitting with the journal's scope, some editors may provide advice to the authors to submit the paper to a certain journal that is much more relevant for submission.

\section{Publishers}

- $\quad$ Promoting open access publications to increase the capacity of acceptance

- Increasing the number of journals that publish papers with negative results

- $\quad$ Rewarding reviewers using acknowledgement, pre-publication history and other means of motivations.

\section{References}

1. Woolley KL, Barron JP (2009) Handling manuscript rejection: insights from evidence and experience. Chest 135: 573-577.

2. Turcotte C, Drolet P, Girard M (2004) Study design, originality and overall consistency influence acceptance or rejection of manuscripts submitted to the Journal. Can J Anaesth 51: 549-556.

3. Ajao OG (2005) Some Reasons for Manuscript Rejection by PeerReviewed Journals. Annals of Ibadan Postgraduate Medicine 3 : 9-12.
4. Ehara S, Takahashi K (2007) Reasons for rejection of manuscripts submitted to AJR by international authors. AJR Am J Roentgenol 188: W113-116.

5. Calcagno V, Demoinet E, Gollner K, Guidi L, Ruths D, et al. (2012) Flows of research manuscripts among scientific journals reveal hidden submission patterns. Science.

6. Murray DM, Pals SL, Blitstein JL, Alfano CM, Lehman J (2008) Design and analysis of group-randomized trials in cancer: a review of current practices. J Natl Cancer Inst 100: 483-491.

7. Kelly KD, Travers A, Dorgan M, Slater L, Rowe BH (2001) Evaluating the quality of systematic reviews in the emergency medicine literature. Ann Emerg Med 38: 518-526.

8. Norman G (2010) Likert scales, levels of measurement and the "laws" of statistics. Adv Health Sci Educ Theory Pract 15: 625-632.

9. Dutta MJ (2006) The ten commandments of reviewing: the promise of a kinder, gentler discipline! Health Commun 20: 197-200.

10. Smith R (2006) Peer review: a flawed process at the heart of science and journals. J R Soc Med 99: 178-182.

11. Lee KP, Boyd EA, Holroyd-Leduc JM, Bacchetti P, Bero LA (2006) Predictors of publication: characteristics of submitted manuscripts associated with acceptance at major biomedical journals. The Medical Journal of Australia 184: 621-626. 